Relations industrielles

Industrial Relations

\title{
Reuther, par Frank Cormier et William J. Eaton, Englewood Cliffs, N.J., Prentice-Hall, Inc., 1970, 475 pp.
}

\section{Gérard Dion}

Volume 28, numéro 4, 1973

URI : https://id.erudit.org/iderudit/028471ar

DOI : https://doi.org/10.7202/028471ar

Aller au sommaire du numéro

Éditeur(s)

Département des relations industrielles de l'Université Laval

ISSN

0034-379X (imprimé)

1703-8138 (numérique)

Découvrir la revue

Citer ce compte rendu

Dion, G. (1973). Compte rendu de [Reuther, par Frank Cormier et William J. Eaton, Englewood Cliffs, N.J., Prentice-Hall, Inc., 1970, 475 pp.] Relations industrielles / Industrial Relations, 28(4), 900-901.

https://doi.org/10.7202/028471ar

Tous droits réservés @ Département des relations industrielles de l'Université Laval, 1973
Ce document est protégé par la loi sur le droit d'auteur. L’utilisation des services d'Érudit (y compris la reproduction) est assujettie à sa politique d'utilisation que vous pouvez consulter en ligne.

https://apropos.erudit.org/fr/usagers/politique-dutilisation/ 
Selected Models of Worker Education in Europe, Research Series III, by Robert E. Beldings, Iowa City, The University of Iowa, Center for Labor and Management, 1973, $142 \mathrm{pp}$.

This collection of articles, all written by the editor, provides a broad transnational view of the development, structure and techniques of adult education. Its specific intent is to challenge its readers to scrutinize the major European models of worker education and hopefully borrow that which is relevant and workable within their own economics. The volume includes eighteen articles: twelve of these were originally published in journals of education within the past decade.

The initial article outlines the development and structure of the French vocational education system, one of the earliest efforts in this direction. Worker education in England is covered in the next three articles. Topics include the Cadbury institutions for secondary schooling, the model adult education programs of the Workers' Education Association, and the experimental approach of the Open University. The Soviet Union is then briefly treated in an essay on its highly centralized and comprehensive educational programs.

One of the lengthier works in this collection describes the Geneva based Organization for Rehabilitation through Training (ORT). The author traces its evolution from a Jewish welfare effort to a complex institution with major ongoing programs in France, the Middle East, Africa and Latin America. A detailed view is provided of ORT's manpower surveys and centres for program development and teacher trainings.

Germany's significant influence on worker education is described in three articles. One deals with leadership college of the German labour federation (DGB), while the others outline the emergence of that country's frequently emulated models of vocational education.

The remaining eight articles are devoted to the Scandinavian experience with vocational training, retraining and adult continuing education. After a short introductory essay, three works describe the Danish folk high school and the programs of the Danish Workers'
Education Association. The next pair of articles present short treatments of Sweden's manpower training institutions and the popular study circles. Attention is then directed toward Norway's system of vocational education and the recently developed district colleges. The last piece in the series concerns itself with Finland's approach to population shifts and changing manpower requirements.

The author concludes his anthology by comparing the numerous models on a general level, carefully avoiding the temptation of making any sweeping prescriptions for resolving North America's manpower problems. His brief and well written articles manage to cover a wide variety of worker education programs and techniques. It might be reasonably argued that too heavy an emphasis has been placed on Scandinavian approaches or that the international etfort of ORT has been misclassified as a European model. Nevertheless the author succeeds in presenting a stimulating overview of worker education within a cross cultural framework.

University of New Brunswick

Gary N. CHAISON

Reuther, par Frank Cormier et William J. Eaton, Englewood Cliffs, N.J., Prentice-Hall, Inc., 1970, 475 pp.

Walter Reuther a certainement été un des dirigeants syndicaux américains dont la personnalité et le caractère ont marqué l'évolution du syndicalisme dans ce pays depuis l'époque de Roosevelt avec son New Deal.

Dans cette biographie, les auteurs retracent la vie du personnage depuis son enfance jusqu'à sa mort prématurée en 1970. Ils montrent le jeune Walter dans son milieu familial où se développe déjà sa vocation syndicale. Ils permettent de le suivre dans ses premières expériences de travailleur et, à la suite de son coup de tête, dans son tour d'Europe qui le conduit à aller travailler dans une usine en Russie communiste. A son retour aux Etats-Unis, c'est alors toute sa carrière de dirigeant syndical qui est racontée en le suivant comme organisateur d'abord réussissant à briser la résistance patronale et à implanter le syndicalisme chez General Motors à Flint et ensuite gravissant la hiérarchie 
jusqu'à accéder à la présidence des United Automobile Workers. Reuther socialiste doit mener la lutte au sein de son syndicat contre les communistes qui voulaient en prendre le contrôle. On le voit dans tous les combats qu'il a menés tant contre l'industrie géante qu'au sein du mouvement syndical et qu'au plan politique. On le voit aussi dans ses rapports avec des personnages importants avec qui il a eu à traiter au cours de sa carrière : John L. Lewis, Philip Murray, George Meany, Sidney Hillman, Khrushchev et les présidents Roosevelt, Truman, Kennedy, Johnson. Pour Reuther, le syndicalisme n'était pas simplement une agence des services au profit de ses membres, il avait un rôle économique, social et politique à jouer tant au niveau national qu'international.

Le texte est chargé de citations de déclaratoins et de discours prononcés par Reuther qui illustrent et confirment ses positions dans les divers moments importants de son activité syndicale et les luttes qu'il a eu à livrer sur tous les terrains. Le fait que les références bibliographiques soient reléguées dans un annexe consacré aux sources soulage le lecteur et en même temps satisfait le chercheur qui veut vérifier ou pousser plus loin les études sur une situation particulière.

Les auteurs ont procédé d'une façon beaucoup plus anecdotique qu'analytique et critique. Ils ont présenté un ouvrage sympathique, mais honnête, qui est de lecture facile, même pour ceux qui ne sont pas très familiers avec le monde syndical.

Université Laval

Gérard DION

Canadian Cases in Labour Relations and Collective Bargaining, by Hem C. Jain, Don Mills (Ontario), Longman Canada Limited, 1973, 211 pp.

L'auteur de cet ouvrage entend combler un vide dans la littérature pédagogique en relations industrielles au Canada. En effet, le professeur qui veut illustrer son enseignement à l'aide d'exemples vécus ou qui veut susciter chez ses étudiants des discussions autour de situations réelles ne dispose pas de recueil de cas proprement canadien dans le domaine des relations du travail. Il doit la plupart du temps se référer à des ouvrages américains ou repérer luimême les cas qu'il entend utiliser ou repérer.

Or, même si l'on observe certains points de ressemblance entre les cadres américain et canadien de relations du travail, il demeure que le régime canadien comporte des caractéristiques qui lui sont propres de telle sorte que les problèmes ne se posent pas nécessairement de la même façon pas plus qu'ils ne donnent lieu aux mêmes solutions.

C'est à partir de cette observation que le professeur Jain a rassemblé un certain nombre de 《cas 》 canadiens qu'il propose dans ce recueil. Ces derniers, choisis dans diverses juridictions provinciales et fédérale, sont regroupés sous quatre titres : la détermination des parties à la négociation, la négociation comme telle, la négociation de quelques questions majeures (telles que l'ancienneté, les droits de la direction, la sous-traitance, les changements technologiques, etc.) et la coopération patronale-syndicale.

Chaque cas comprend l'information nécessaire à l'analyse et à la discussion de la situation présentée ; c'est-à-dire une brève description des éléments factuels, les positions syndicale et patronale, un guide de discussion et enfin la décision rendue par une instance compétente (Commission de relations du travail, Tribunal d'arbitrage, Commission d'enquête, etc.) lorsqu'il y a lieu.

L'intérêt de la plupart des cas proposés repose sur le fait qu'ils sont souvent le reflet de situations particulièrement critiques. C'est ainsi, par exemple, que le cas portant sur la question de sous-contrats en est un qui remet en cause l'existence même de l'unité de négociation, celui relatif à l'exercice des droits de la direction réfère à la célèbre cause Port Arthur Shipbuilding, celui sur les changements technologiques s'insère dans le cadre du rapport Freedman, et on pourrait multiplier les exemples.

Même si dans la pratique quotidienne des relations du travail, les parties ne font pas toujours face à des situations limites, la discussion des cas proposés par le professeur Jain pourra sans doute permettre à l'étudiant de mieux découvrir et mettre en lumière les principes en cause dans chacune des situations présentées. 